\title{
A new physical interpretation of optical and infrared variability in quasars
}

\author{
Nicholas P. Ross, ${ }^{1 \star}$ K. E. Saavik Ford, ${ }^{2,3,4}$ Matthew Graham, ${ }^{5}$ Barry McKernan, $, 3,4$ \\ Daniel Stern, ${ }^{6}$ Aaron M. Meisner, ${ }^{7,8}$ Roberto J. Assef, ${ }^{9}$ Arjun Dey, ${ }^{10}$ \\ Andrew J. Drake, ${ }^{11}$ Hyunsung D. Jun ${ }^{12}$ and Dustin Lang ${ }^{13,14,15}$ \\ ${ }^{1}$ Institute for Astronomy, University of Edinburgh, Royal Observatory, Blackford Hill, Edinburgh EH9 3HJ, UK \\ ${ }^{2}$ Department of Science, BMCC, City University of New York, New York, NY 10007, USA \\ ${ }^{3}$ Department of Astrophysics, Rose Center for Earth and Space, American Museum of Natural History, Central Park West at 79th Street, NY 10024, USA \\ ${ }^{4}$ Graduate Center, City University of New York, 365 5th Avenue, New York, NY 10016, USA \\ ${ }^{5}$ Cahill Center for Astronomy and Astrophysics, California Institute of Technology, Mail Code 249/17, 1200 E California Blvd, Pasadena, CA 91125, USA \\ ${ }^{6}$ Jet Propulsion Laboratory, California Institute of Technology, 4800 Oak Grove Drive, Mail Stop 169-221, Pasadena, CA 91109, USA \\ ${ }^{7}$ Lawrence Berkeley National Laboratory, 1 Cyclotron Road, Berkeley, CA 92420, USA \\ ${ }^{8}$ Berkeley Center for Cosmological Physics, Berkeley, CA 94720, USA \\ ${ }^{9}$ Núcleo de Astronomía de la Facultad de Ingeniería y Ciencias, Universidad Diego Portales, Av. Ejército Libertador 441, Santiago, Chile \\ ${ }^{10}$ National Optical Astronomy Observatory, 950 N. Cherry Ave, Tucson, AZ 85719, USA \\ ${ }^{11}$ Center for Advanced Computing Research, California Institute of Technology, 1200 E California Blvd, Pasadena, CA 91125, USA \\ ${ }^{12}$ School of Physics, Korea Institute for Advanced Study, 85 Hoegiro, Dongdaemun-gu, Seoul 02455, Korea \\ ${ }^{13}$ Dunlap Institute, University of Toronto, Toronto, ON M5S 3H4, Canada \\ ${ }^{14}$ Department of Astronomy \& Astrophysics, University of Toronto, Toronto, ON M5S 3H4, Canada \\ ${ }^{15}$ Perimeter Institute for Theoretical Physics, Waterloo, ON N2L 2Y5, Canada
}

\begin{abstract}
Changing-look quasars are a recently identified class of active galaxies in which the strong UV continuum and/or broad optical hydrogen emission lines associated with unobscured quasars either appear or disappear on time-scales of months to years. The physical processes responsible for this behaviour are still debated, but changes in the black hole accretion rate or accretion disc structure appear more likely than changes in obscuration. Here, we report on four epochs of spectroscopy of SDSS J110057.70-005304.5, a quasar at a redshift of $z=0.378$ whose UV continuum and broad hydrogen emission lines have faded, and then returned over the past $\approx 20 \mathrm{yr}$. The change in this quasar was initially identified in the infrared, and an archival spectrum from 2010 shows an intermediate phase of the transition during which the flux below rest frame $\approx 3400 \AA$ has decreased by close to an order of magnitude. This combination is unique compared to previously published examples of changing-look quasars, and is best explained by dramatic changes in the innermost regions of the accretion disc. The optical continuum has been rising since mid-2016, leading to a prediction of a rise in hydrogen emission-line flux in the next year. Increases in the infrared flux are beginning to follow, delayed by a $\sim 3 \mathrm{yr}$ observed time-scale. If our model is confirmed, the physics of changing-look quasars are governed by processes at the innermost stable circular orbit around the black hole, and the structure of the innermost disc. The easily identifiable and monitored changing-look quasars would then provide a new probe and laboratory of the nuclear central engine.
\end{abstract}

Key words: accretion, accretion discs-surveys-quasars: general-quasars: individual: J1100-0053. 


\section{INTRODUCTION}

The Shakura-Sunyaev $\alpha$-disc model (Shakura \& Sunyaev 1973) has long been used to describe the basic properties of the optically thick, geometrically thin accretion discs expected to orbit the supermassive black holes at the nuclei of quasars. This accretion disc is thought to be the origin of thermal continuum emission observed in the rest-frame ultraviolet (UV) and optical. The thermal emission seen in the infrared (IR) spectrum of quasars is believed to originate from molecular dust outside the accretion disc and traditional broad-line region (BLR). Thus, the IR flux is directly proportional to the emission from the disc, reprocessed by the dusty reservoir and delayed by the light-travel time between the two (see e.g. Antonucci 1993; Perlman et al. 2008; Lasota 2016, for reviews). As such, the thermal accretion disc photons are the seeds for both the X-ray emission - due to Compton-upscattering in the corona (e.g. Begelman, McKee \& Shields 1983; Risaliti, Young \& Elvis 2009; Lusso \& Risaliti 2017) and the thermal mid-IR emission from the torus.

The $\alpha$-disc model assumes that the disc is geometrically thin (i.e. $h / r \ll 1$, where $h / r$ is the disc aspect ratio) and that angular momentum is transported by a kinematic viscosity, $v$, parametrized by Shakura \& Sunyaev (1973) as

$v=\alpha c_{\mathrm{s}} h$,

where $c_{\mathrm{s}}$ is the local mean sound speed in the disc and $h$ is the scale height perpendicular to the disc plane. The thermal emission needs not be, but often is, treated as a superposition of blackbodies at varying annuli with an effective temperature dependence ${ }^{1}$ going as $T(r) \propto r^{-3 / 4}$.

Given the size scales and temperatures associated with supermassive black holes, a substantial fraction of the bolometric luminosity should be in the form of UV photons - the so-called Big Blue Bump (Shields 1978; Malkan \& Sargent 1982). For the optically thick, UV emitting disc to accrete on to the black hole, substantial angular momentum must be lost. The kinematic viscosity of the plasma, $\alpha$, seems the likely mechanism that transports angular momentum outwards. This viscosity is likely due to magnetorotational instability (Balbus \& Hawley 1991) with additional contributions to turbulence from the effects of objects embedded in the disc (e.g. McKernan et al. 2014).

However, as has long been established (e.g. Alloin et al. 1985) and recently re-visited (e.g. LaMassa et al. 2015; MacLeod et al. 2016; Ruan et al. 2016; Runnoe et al. 2016; Yang et al. 2017; Lawrence 2018; Rumbaugh et al. 2018), the observation of even slowly varying Balmer emission lines in quasars strongly suggests that if a thermal accretion disc does indeed contribute substantially to the ionizing or optical continuum, then it cannot be in quasi-steady-state equilibrium. The variations must be due to more chaotic disturbances or instabilities in the disc that propagate at considerably higher speeds than the radial accretion flow and possibly as fast as the orbital velocity.

Furthermore, as e.g. Koratkar \& Blaes (1999) and Sirko \& Goodman (2003) among others point out, the observed spectral energy distributions (SEDs) of typical quasars differ markedly from classical $\alpha$-disc theoretical predictions (Shakura \& Sunyaev 1973; Pringle
1981) with a typical observed quasar SED flat in $\lambda F_{\lambda}$ over several decades in wavelength (Elvis et al. 1994; Richards et al. 2006). Also, real active galactic nucleus (AGN) discs seem to be cooler (e.g. Lawrence 2012) and larger (e.g. Pooley et al. 2007; Morgan et al. 2010; Mosquera \& Kochanek 2011; Morgan et al. 2012) than the $\alpha$-disc model predicts. Furthermore, the $\alpha$-disc is an ad hoc parametrization of disc viscosity and does not permit predictions of global changes from local perturbations (King 2012).

Nevertheless, in this paper, we utilize the mathematically simple $\alpha$-disc model as a framework and departure point for our own disc models. Here, we build on previous work (Sirko \& Goodman 2003; Zimmerman et al. 2005; Hameury, Viallet \& Lasota 2009) and introduce a phenomenological model that does allow changes across the accretion disc and, crucially, makes predictions that can be observed in the SED.

Changing-look quasars (CLQs) are luminous active galaxies in which the strong UV continuum and/or broad optical hydrogen emission lines associated with unobscured quasars either appear or disappear on time-scales of months to years. CLQs have traditionally been discovered by looking for large, $|\Delta m|>1$ mag changes in the optical light curves of quasars or galaxies. In contrast, we have taken advantage of the ongoing mid-IR Near-Earth Object Wide-field Infrared Survey Explorer Reactivation (NEOWISE-R) mission (Mainzer et al. 2014), supplemented with the optical Dark Energy Camera Legacy Survey (DECaLS ${ }^{2}$ ), in order to discover new CLQs. While previous efforts have used the $1 \mathrm{yr}$ baseline of the Wide-field Infrared Survey Explorer (WISE) mission to identify CLQs (e.g. Assef et al. 2018; Stern et al. 2018), our investigation is the first to extend this selection to the IR using NEOWISE-R mission data. We have identified a sample of Sloan Digital Sky Survey (SDSS) quasars that show significant changes in their IR flux over the course of a few years. Importantly, our IR light curves enable us to set limits on SED changes due to obscuration.

In this article, we present the $z=0.378$ quasar SDSS J1 10057.70005304.5 (hereafter J1100-0053). J1100-0053 was a known quasar we identified as interesting due to its IR light curve. We have spectral observations for J1100-0053 showing a transition in the blue continuum into a 'dim state', where the rest-frame UV flux is suppressed, and then returning to a state with a strong blue continuum. The model we present invokes changes at the innermost stable circular orbit (ISCO; defined as $r_{\text {ISco }}=\frac{6 G M}{c^{2}}$ in the Schwarzschild metric) to be the triggering event for substantial changes in the wider accretion disc, including major structural changes out to $\approx 225 r_{\mathrm{g}}$ (where $r_{\mathrm{g}}$ is the gravitational radius; $r_{\mathrm{g}}=\frac{G M}{c^{2}}$ ). Such a model explains changes in the broad emission lines, as well as the optical and IR light curves.

This paper is organised as follows. In Section 2, we describe our sample selection, catalogues and observation data sets. In Section 3, we present various theoretical models and discuss if and how each describes and explains the data. We conclude in Section 4. We report all magnitudes on the $\mathrm{AB}$ zero-point system (Oke \& Gunn 1983; Fukugita et al. 1996) unless otherwise stated explicitly. For the WISE bands, $m_{\mathrm{AB}}=m_{\text {Vega }}+m$ where $m=(2.699,3.339)$ for WISE $W 1$ at $3.4 \mu \mathrm{m}$ and WISE $W 2$ at $4.6 \mu \mathrm{m}$, respectively (Cutri et al. 2011). We choose the cosmological parameters $\Omega_{\Lambda}=0.7$, $\Omega_{\mathrm{M}}=0.3$, and $h=0.7$ in order to be consistent with Shen et al. (2011).

\footnotetext{
${ }^{1}$ This $r^{-3 / 4}$ temperature dependence is not specific to the $\alpha$-parametrization and is independent from the nature of the viscosity, provided that the disc is geometrically thin, steady, with heat dissipation and angular momentum transport caused by the same, local mechanism.
}

${ }^{2}$ legacysurvey. org/decamls / 


\section{TARGET SELECTION AND OBSERVATIONS}

\subsection{Selection in SDSS and NEOWISE-R of J1100-0053}

We started by matching the SDSS Data Release 7 quasar (DR7Q; Schneider et al. 2007) and the SDSS-III Baryon Oscillation Spectroscopic Survey (BOSS) Data Release 12 quasar (DR12Q) catalogues (Pâris et al. 2017) to the NEOWISE-R IR data. We use data from the beginning of the WISE mission (2010 January; Wright et al. 2010) through the third year of NEOWISE-R operations (2016 December; Mainzer et al. 2011). The WISE scan pattern leads to coverage of the full-sky approximately once every six months (a 'sky pass'), but the satellite was placed in hibernation in 2011 February and then reactivated in 2013 October. Hence, our light curves have a cadence of 6 months with a 32 month sampling gap.

The $W 1 / W 2$ light curves for $\sim 200000$ SDSS and BOSS spectroscopic quasars were obtained by performing forced photometry at the locations of DECam-detected optical sources (Lang 2014; Meisner et al. 2017a; Meisner, Lang \& Schlegel 2017b). This forced photometry was performed on time-resolved co-adds (Lang 2014), each of which represents a stack of $\sim 12$ exposures. The co-addition removes the possibility of probing variability on $\lesssim 1 \mathrm{~d}$ time-scales, but pushes $\approx 1.4$ mag deeper than individual exposures while removing virtually all single-exposure artefacts (e.g. cosmic rays and satellites).

Approximately, $\sim 30000$ of the SDSS/BOSS quasars with $W 1 / W 2$ light curves available are 'IR bright', in that they are above both the $W 1$ and $W 2$ single-exposure thresholds and therefore detected at very high significance in the co-adds. For this ensemble of objects, the typical variation in each quasar's measured (W1-W2) colour is $0.06 \mathrm{mag}$. This includes statistical and systematic errors that are expected to contribute variations at the few hundredths of a magnitude level. The typical measured single-band scatter is 0.07 mag in each of $W 1$ and $W 2$.

We undertook a search for outliers relative to these trends. Specifically, we selected objects with the following characteristics:

(i) Monotonic variation in both $W 1$ and $W 2$ flux.

(ii) $W 1$ flux and $W 2$ flux Pearson correlation coefficient $r \geq 0.9$. (iii) $>0.5$ mag peak-to-peak variation in either $W 1$ or $W 2$.

This yields a sample of 248 sources. 31 of these are assumed to be blazars due to the presence of Faint Images of the Radio Sky at Twenty-Centimeters (FIRST; Becker, White \& Helfand 1995) radio counterparts, and we discount them for further analyses. Another 22 objects are outside the FIRST footprint, leaving 195 quasars in our IR-variable sample, with no potential FIRST counterparts detected within 30 arcsec.

Although aperture photometry and DECaLS forced photometry (Lang 2014; Meisner et al. 2017a,b) are available, J1100-0053 is significantly above the single-exposure detection limit, so it is valid to obtain photometry from the publicly released $W 1 / W 2$ Level $1 b$ (L1b) single-exposure images at the NASA/IPAC Infrared Science Archive (http://irsa.ipac.caltech.edu/IRSA). Upon querying the combined the WISE All-Sky, WISE Post-Cryo and NEOWISE$\mathrm{R}$ data bases, we have 101 measurements in 8 sky passes spanning nearly $2400 \mathrm{~d}$.

Links to all our data, catalogues and analysis can be found online at: https://github.com/d80b2t.

\subsection{Optical imaging}

Fig. 1 presents the light curve of SDSS J1100-0053. J1100-0053 was first detected in the National Geographic Society-Palomar Observatory Sky Survey (NGS-POSS; Abell 1959; Minkowski \& Abell 1963 ) in 1955 April. It is catalogued in the SuperCOSMOS Science Archive (http://ssa.roe.ac.uk/SSA; Hambly et al. 2001a; Hambly, Irwin \& MacGillivray 2001b) and due to its equatorial position was also observed by the UK Schmidt Telescope (UKST; Cannon 1975, 1979). Querying the SSA returns gCorMag and sCorMag that are the magnitudes assuming the object is either a galaxy or star, respectively. We use the sCorMag values as is appropriate for an image with flux dominated by the point-like AGN; the $s$ CorMag magnitudes are calibrated in the Vega system. For J1100-0053, we find the magnitudes are $18.10 \mathrm{mag}$ in the blue UK-J filter from MJD 45440.47 (1983 April 16); 17.49 mag in the red POSS-I 'E'filter from MJD 35214.22 (1955 April 17); $17.92 \mathrm{mag}$ in the red UK-R filter from MJD 46521.47 (1986 April 01) and $17.71 \mathrm{mag}$ in the UK-I filter from MJD 47273.49 (1988 April 22). J1100-0053 is not in the Digital Access to a Sky Century @ Harvard $\left(\mathrm{DASCH}^{3}\right)$.

$\mathrm{J} 1100-0053$ was imaged by the SDSS in the $u, g, r, i$, and $z$ bands in 1999 March, and more recently by the DECaLS, where there are 4,13 , and 4 exposures in the $g, r$, and $z$ bands, respectively, in the DECaLS Data Release 3 (DR3; Dey et al. 2018). The $g$-band observations span $\approx 3 \mathrm{yr}\left(56727 \leq g_{\text {MJD }} \leq 57816\right)$, while the $r$ and $z$-band observations span $\approx 4 \mathrm{yr}\left(56367 \leq r_{\mathrm{MJD}} \leq 57814\right.$ and $\left.56383 \leq z_{\mathrm{MJD}} \leq 57815\right)$.

Along with WISE IR data, optical data from the SDSS, Catalina Real-time Transient Survey (CRTS; Drake et al. 2009; Mahabal et al. 2011), the Lincoln Near-Earth Asteroid Research (LINEAR; Sesar et al. 2011) programme, and the Panoramic Survey Telescope and Rapid Response System (PanSTARRS; Kaiser et al. 2010; Stubbs et al. 2010; Tonry et al. 2012; Magnier et al. 2013) are also available, and presented in Fig. 1.

\subsection{Additional multiwavelength data for J1100-0053}

J1100-0053 was observed by Röntgen satellite (ROSAT) and appears in the All-Sky Survey Bright Source Catalogue (RASSBSC; Appenzeller et al. 1998; Voges et al. 1999) as 2RXS J110058.1-005259 with 27.00 counts (count error 6.14) and a count rate $=0.06 \pm 0.01$ counts s $^{-1}$ (Boller et al. 2016). The NASA/IPAC Extragalactic Database (NED ${ }^{4}$ ) gives J1100-0053 as having a flux of $1.27 \pm 0.28 \times 10^{-12} \mathrm{erg} \mathrm{cm}^{-2} \mathrm{~s}^{-1}$ in the $0.1-2.4 \mathrm{keV}$ range (unabsorbed). J1100-0053 is not in either the Chandra or XMM-Newton archives but is detected by the Galaxy Evolution Explorer (GALEX; Martin et al. 2005; Morrissey et al. 2007) and has reported flux densities of $19.29 \pm 0.12 \mathrm{mag}$ in the far-UV and $18.89 \pm 0.05 \mathrm{mag}$ in the near-UV. As noted above, there is no radio counterpart within 30 arcsec in the FIRST survey, i.e. at $21 \mathrm{~cm}$. None of the Hubble Space Telescope, Spitzer Space Telescope, or Kepler missions have observed J1100-0053. It is also not in the Hyper Suprime-Cam (HSC) Data Release 1 (Aihara et al. 2018) footprint.

\subsection{Spectroscopy}

\subsubsection{SDSS and BOSS spectroscopy}

Fig. 2 shows four optical spectra of J1100-0053. J1100-0053 satis-

${ }^{3}$ http://dasch.rc.fas.harvard.edu/project.php

${ }^{4}$ https://ned.ipac.caltech.edu/ 


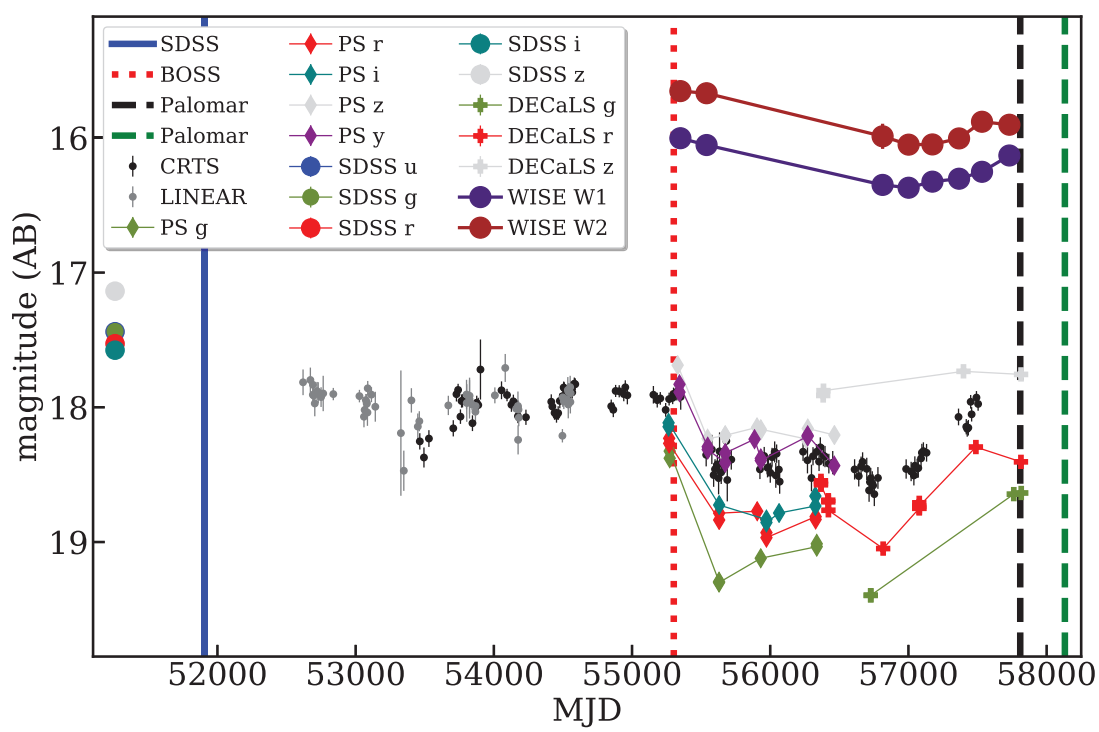

Figure 1. Multiwavelength light curve of J1100-0053, including optical data from LINEAR, CRTS, SDSS, PanSTARRS, and DECaLS, and mid-IR data from the WISE satellite. The four vertical lines illustrate the four epochs of optical spectra presented in Fig. 2. J1100-0053 was flagged for further study due to the IR fading observed by WISE. Note that the optical emission has been recovering over the past few years, with the IR emission beginning to show similar behaviour.

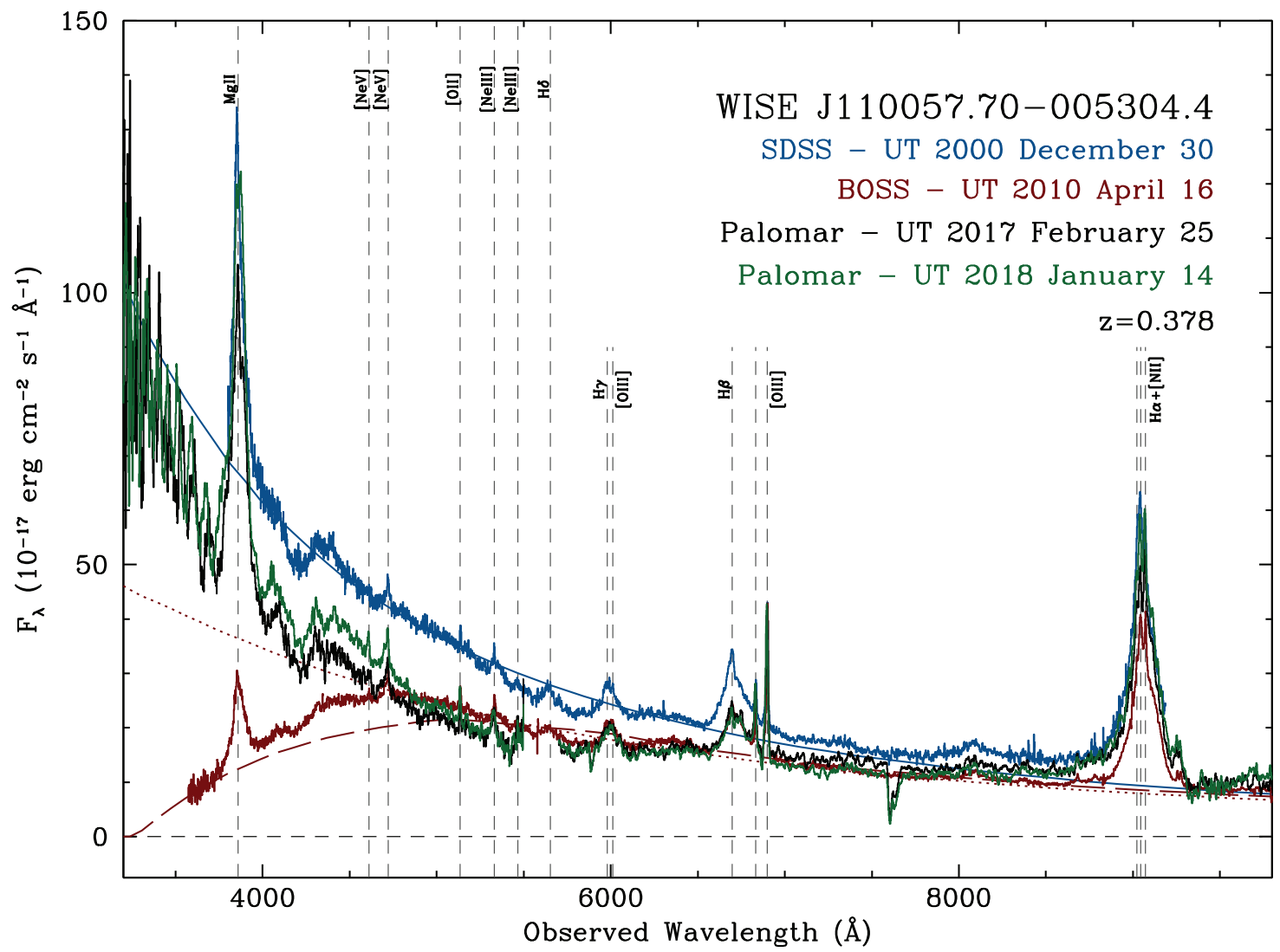

Figure 2. Optical spectra of J1100-0053 obtained on MJD 51908 (blue; SDSS), 55302 (red; BOSS), 57809 (black; Palomar), and 58132 (green; Palomar). Spectra have been renormalized to maintain a constant [O III] luminosity. Over the past two decades, the UV continuum and broad lines have changed significantly for this quasar. In particular, the second-epoch BOSS spectrum from 2010 shows the rare occurrence of a temporary collapse of the UV continuum. The smooth lines show three simple thermal accretion disc models of the continuum. As discussed in Section 3, torque at the ISCO, possibly due to magnetic fields threading the inner disc and plunging region, heats the inner disc, causing it to puff up and become more UV luminous. The solid blue line shows an inflated disc with non-zero torque at the ISCO. The dotted red line shows a modified zero-torque model where the thermal disc emission interior to $40 r_{\mathrm{g}}$ is suppressed by a factor of 10. The red long dashed line is a zero torque at the ISCO model multiplied by an absorption law adapted from Guo et al. (2016). 
fied a number of spectroscopic targeting flags, making it a quasar target in SDSS (Richards et al. 2002). An SDSS spectrum was obtained on MJD 51908 (SDSS plate 277, fibre 212) and the spectrum of a $z=0.378$ quasar was catalogued in the SDSS Early Data Release (Schneider et al. 2002; Stoughton et al. 2002).

The second-epoch spectrum is from the SDSS-III BOSS (Dawson et al. 2013) on MJD 55302 and shows the downturn at $\lesssim 4300 \AA$ (observed). SDSS-III BOSS actively vetoed previously known $z<$ 2 quasars (Ross et al. 2012), but due to J1100-0053 being selected as an ancillary target (via a white dwarf programme; Kepler et al. 2015,2016 ), a second spectral epoch was obtained. Due to a design trade-off to improve throughput in the Ly $\alpha$ forest of quasar spectra in BOSS, quasar targets were subject to spectrophotometric calibration errors (Margala et al. 2016). These are introduced primarily due to offsets in fibre-hole positioning between quasar targets and spectrophotometric standard stars. However, since J1100-0053 was not a BOSS quasar target, it is not subject to this 'blue offset'. $\mathrm{J} 1100-0053$ has no pipeline flag suggesting the spectrum was compromised during data taking. We checked the calibration of BOSS Plate 3836 that observed J1100-0053 and confirmed that the data were high-SNR and that the behaviour in the blue spectrum was not due to the instrument, telescope, or data reduction.

One significant aspect of the BOSS spectrum is the strong, broad Mg II emission line atop a fading red continuum. $\mathrm{Mg}_{\text {II }}$ emission being prominent in objects without otherwise strong blue continuum is very unusual, but not unheard of. For example, Roig, Blanton \& Ross (2014) find a group of objects with strong and broad Mg II line emission, but very weak $\mathrm{H} \alpha$ and $\mathrm{H} \beta$ emissions, and undetectably low near-UV AGN continuum flux.

\subsubsection{Palomar spectroscopy}

A third-epoch spectrum was obtained from the Palomar Hale $5 \mathrm{~m}$ telescope using the Double Spectrograph (DBSP) instrument. Exposures of 600 and $300 \mathrm{~s}$ were taken in good conditions on UT 2017 February 25 (MJD 57809). Features to note include the continuum straddling $\mathrm{Mg}$ II being blue in the 2017 spectrum, as it was for the SDSS spectrum in 2000, as opposed to red, as it was for the BOSS spectrum in 2010. A fourth spectral epoch was also taken using the Hale $5 \mathrm{~m}$ and DBSP on UT 2018 January 14 (MJD 58132).

The first-epoch SDSS spectrum shows a typical blue quasar, but the blue continuum decreases by nearly a factor of ten in flux in the second-epoch BOSS spectrum taken $10 \mathrm{yr}$ later. The blue continuum then returns in the third-epoch spectrum taken another $7 \mathrm{yr}$ later, albeit at a diminished level relative to the initial spectrum. The final spectrum obtained, approximately $1 \mathrm{yr}$ late, is similar to the third-epoch spectrum.

The SDSS and BOSS spectroscopy is performed with 3 and 2 arcsec fibres, respectively. The Palomar observations are done with 1.5 arcsec slits. We note that the 2017 Palomar data were photometric, while 2018 Palomar data were not. Both Palomar spectra were flux calibrated using the sensitivity function calculated from the photometric night (in 2017), and then the spectra were scaled to have approximately matching $\left[\mathrm{O}_{\mathrm{III}}\right]$ line strengths. As such, in the following, we note that the larger [O III] luminosities obtained with the fibre spectra as opposed to the slit spectra is consistent with [O III] being obtained through wider apertures and the line being somewhat extended.

\subsubsection{Emission-line fitting}

We fit the SDSS spectrum for J1100-0053 with the models provided by QSFit $^{5}$ (Calderone et al. 2017) and require three components in order to replicate the observed asymmetric $\mathrm{H} \beta$ emission-line profile: a narrow full width at half-maximum $(F W H M)=1000 \mathrm{~km} \mathrm{~s}^{-1}$ component, and two broad (11 $897 \pm 556$ and $\left.4594 \pm 341 \mathrm{~km} \mathrm{~s}^{-1}\right)$ components. The two broad components overlap and hence QSFit consider them as a single line, whose profile is given by the sum of the two Gaussians. The final FWHM of $7415 \pm 499 \mathrm{~km} \mathrm{~s}^{-1}$ is calculated on the latter profile. Thus, we suggest that J1100-0053, at least from its SDSS epoch, could be an 'anomalous $\mathrm{H} \beta$ quasar' as described by Steinhardt \& Silverman (2013). Those authors suggest that $\sim 1 / 4$ of all SDSS quasars at $z<0.8$ for which $\mathrm{H} \beta$ is well measured is best described by not one, but two well-centred broad components, resulting in the 'anomalous' broadened $\mathrm{H} \beta$ lines.

Using the methods presented in Jun et al. (2015a), we perform further fits to the $\mathrm{H} \beta$ and [O III] line complex and the results are given in Table 1. The total $\mathrm{H} \beta$ (with 2 broad +1 narrow components) to total $[\mathrm{O} \mathrm{III}]$ ( 1 broad+1 narrow, where the narrow width/centre is fixed to the Balmer components) ratio drops by a factor $\times 2.3$ times and $1.5,1.9$ times in the third and fourth epochs. The $5100 \AA$ continuum to total $[\mathrm{O}$ III] ratios also change by $\times 1.9,1.7$, and 2.1 in the second, third, and fourth epochs, indicating that the $5100 \AA$ and $\mathrm{H} \beta$ luminosities vary consistently. We return to the $\mathrm{H} \beta$ line profile in our modelling discussion in Section 3.

While continuum changes in the rest-frame UV/optical spectra of quasars are not a new discovery (see e.g. Clavel et al. 1991, the review by Ulrich, Maraschi \& Urry 1997 and more recent studies by Vanden Berk et al. 2004; Pereyra et al. 2006; MacLeod et al. 2010 and Guo \& Gu 2016), the identification of a 'UV collapse' for quasars has only recently been noted by Guo et al. (2016). Those authors report the first discovery of an UV cut-off quasar, SDSS J231742.60+000535.1 (hereafter J2317+0005; redshift $z=0.321$ ), observed spectroscopically by SDSS three times, on UT 2000 September 29, UT 2001 September 25, and UT 2001 October 18. In the case of J2317+0005, a cycle of UV emission collapse, quasar dimming, and recovery was observed over the course of just a few weeks. For J1100-0053, the cycle is far longer; however, the combination of optical and IR light curves, as well as observing J1100-0053 at four separate spectral stages is currently unique. As such, J1100-0053 and J2317+0005 are now two archetypal objects that any accretion disc model must predict and explain (e.g. Lawrence 2018).

In our companion study, Stern et al. (2018) report on a new CLQ, J1052+1519, identified with the same selection as J11000053, and where the broad $\mathrm{H} \beta$ emission has vanished compared to an archival SDSS spectrum. The physical properties of J1100-0053 derived from the MJD 51908 spectrum using the methods in Shen et al. (2011), are given in Table 2, where we also give the properties of J2317+0005 (Guo et al. 2016) and J1052+1519 (Stern et al. 2018) for comparison.

\section{PHENOMENOLOGICAL MODELLING}

In a similar vein to the discussion in Stern et al. (2018), in this section we discuss several models with the aim of determining the physical mechanism (s) driving the light curve and spectral behaviour of J1100-0053. The explanations come in two broad

\footnotetext{
${ }^{5}$ http://qsfit.inaf.it/
} 
Table 1. Spectral values to the $\mathrm{H} \beta\left[\mathrm{O}\right.$ III] line complex using the methods of Jun et al. (2015a). FWHM in $\mathrm{km} \mathrm{s}^{-1}$ and luminosities are in $\mathrm{erg} \mathrm{s}^{-1}$. The final two rows give the ratio of the total $\mathrm{H} \beta$ to total [O III] luminosities, and total 5100 Å continuum to total [O III] line luminosities, respectively.

\begin{tabular}{lcccc}
\hline $\begin{array}{l}\text { Spectrum } \\
\text { MJD }\end{array}$ & SDSS & BOSS & $\begin{array}{c}\text { Palomar } \\
57809\end{array}$ & $\begin{array}{c}\text { Palomar } \\
58132\end{array}$ \\
\hline FWHM H $\beta$ (broad) & 51908 & 55302 & $6684 \pm 180$ & $7657 \pm 239$ \\
$\log \left(\mathrm{BH}\right.$ mass $\left./ \mathrm{M}_{\odot}\right)$ & $8.89 \pm 0.12$ & $8.82 \pm 0.12$ & $8.56 \pm 0.12$ & $8.76 \pm 0.12$ \\
$\log L(5100 \AA)$ & $44.78 \pm 0.01$ & $44.56 \pm 0.01$ & $43.96 \pm 0.04$ & $44.11 \pm 0.02$ \\
$\log L(\mathrm{H} \beta$, broad $)$ & $43.05 \pm 0.02$ & $42.76 \pm 0.02$ & $42.29 \pm 0.02$ & $42.44 \pm 0.03$ \\
$\log L(\mathrm{H} \beta$, narrow $)$ & $41.26 \pm 0.61$ & $41.02 \pm 0.61$ & $40.41 \pm 0.61$ & $40.67 \pm 0.61$ \\
$\log L(\mathrm{H} \beta$, total $)$ & $43.06 \pm 0.02$ & $42.76 \pm 0.02$ & $42.294 \pm 0.02$ & $42.44 \pm 0.03$ \\
$\log L([\mathrm{O}$ III] & $42.20 \pm 0.04$ & $42.29 \pm 0.01$ & $41.61 \pm 0.11$ & $41.86 \pm 0.03$ \\
$\mathrm{H} \beta /[\mathrm{O}$ III & 7.18 & 3.07 & 4.89 & 3.79 \\
$5100 \AA /[\mathrm{O}$ III] & 378 & 203 & 225 & 176 \\
\hline
\end{tabular}

Table 2. Physical properties of J1100-0053, J2317+0005, and J1052+1519 using the methods from Shen et al. (2011).

\begin{tabular}{llll}
\hline Quantity & This paper & Guo et al. (2016) & Stern et al. (2018) \\
\hline SDSS name & J110057.70-005304.5 & J231742.60+000535.1 & J105203.55+151929.5 \\
RA/deg & 165.240463 & 349.42752075 & 163.01480103 \\
Declination/deg & -0.884586 & +0.093091 & 15.32488632 \\
Redshift, $z$ & $0.3778 \pm 0.0003$ & $0.3209 \pm 0.0002$ & $0.3022 \pm 0.0008$ \\
SDSS plate, fibre, MJD & $a^{2} 277,212,51908$ & $a_{382,173,51816}$ & $a_{2483,204,53852}$ \\
& & $679,551,52177$ & - \\
BOSS plate, fibre, MJD & $3836,258,55302$ & - & -22.73 \\
$\left.M_{i}(z=2) / \mathrm{mag}^{-1}\right)$ & -24.48 & -23.65 & $45.07 \pm 0.004$ \\
$\log \left(L_{\mathrm{bol}} / \mathrm{erg} \mathrm{s}^{-1}\right)$ & $45.78 \pm 0.02$ & $45.56 \pm 0.004$ & $8.46 \pm 0.02$ \\
$\log \left(M_{\mathrm{BH}} / \mathrm{M}_{\odot}\right)$ & $8.83 \pm 0.14$ & $8.43 \pm 0.03$ & 3.2 \\
Eddington ratio (per cent) & 7.0 & 10.7 & \\
\hline
\end{tabular}

${ }^{a}$ This spectrum was used to estimate the quantities reported. We use the regular definition of $L_{\mathrm{Edd}}=4 \pi G M m_{\mathrm{p}} c / \sigma_{T}=1.26 \times 10^{38}\left(M / \mathrm{M}_{\odot}\right)$ erg s${ }^{-1}$.

classes: obscuration and changes in the accretion disc. Ultimately, we are forced towards a model of the latter type that combines a cooling front propagating in the accretion disc along with changes in the disc opacity.

\subsection{Scenario I: obscuration by an infalling cloud}

We explore the possibility that an obscuring cloud, or clouds, cause the observed light curve and spectral behaviour of J1100-0053. This explanation is dismissed for the CLQ J0159+0033 in LaMassa et al. (2015) but is the preferred explanation for J2317+0005 in Guo et al. (2016).

In this scenario, the obscuring cloud(s) are required to cross the line of sight. The clouds also need to block most of the inner disc such that the ionizing radiation could not impact on the BLR or the torus for a period of months to years, in order to explain both the IR drop and broad-line disappearance. An explanation of why the light curves 'recover' after a period of $\sim 2500 \mathrm{~d}$ (observed frame) is also required; i.e. why do the light curves not rapidly return to their original flux levels once the obscuring event is over.

Clouds should not typically infall; they need to lose angular momentum if they are drawn from a distribution with Keplerian orbits, and even if they do lose angular momentum, e.g. in a collision with clouds of approximately equal mass, they would likely be either destroyed or no longer coherent. The relevant time-scales here are the free fall and cloud-crushing times. The free fall time-scale is

$t_{\mathrm{ff}} \sim 100 \mathrm{yr}\left(\frac{r}{0.4 \mathrm{pc}}\right)^{3 / 2}\left(\frac{M}{10^{8} \mathrm{M}_{\odot}}\right)^{-1}$ and Kelvin-Helmholtz instabilities would destroy the clouds within the cloud-crushing time (e.g. Nagakura \& Yamada 2008; Hopkins 2013; Shiokawa et al. 2015; Bae \& Woo 2016), given by

$$
t_{\mathrm{cc}} \sim 100 \mathrm{yr}\left(\frac{\rho_{\text {cloud }} / \rho_{\text {medium }}}{10^{6}}\right)^{1 / 2}\left(\frac{r_{\text {cloud }}}{4 \times 10^{10} \mathrm{~km}}\right)\left(\frac{v_{\text {rel }}}{10^{4} \mathrm{~km} \mathrm{~s}^{-1}}\right)^{-1} \text {. }
$$

Thus, even if clouds did infall, they would end up fragmented, which should pollute the inner disc. The dust in the cloud would then be well inside the dust sublimation radius

$R_{\text {dust }} \approx 0.4 \mathrm{pc}\left(\frac{L}{10^{45} \mathrm{erg} \mathrm{s}^{-1}}\right)^{1 / 2}\left(\frac{T_{\text {sub }}}{1500 \mathrm{~K}}\right)^{2.6}$

and so the dust will be destroyed in the $\sim 100 \mathrm{yr}$ free fall from the dust-sublimation region. Hence, one cannot absorb the UV spectrum with dust, since it will have been sublimated well before it arrives at the inner disc.

Given the time interval spanned by the spectroscopic observations ( $\approx 7.7 \mathrm{yr}$ ), it is physically possible that an obscuring cloud, just large enough to block our view of the inner disc region could be in a circular orbit at the sublimation radius; this cloud could then cross its own diameter in approximately $6 \mathrm{yr}$ depressing the UV emission. To check this scenario, we use the standard assumptions from Whittet (1992) with $\rho_{\text {dust }}$ being expressed in terms of $\langle A / L\rangle$, extinction per kpc for the interstellar medium (ISM):

$\rho_{\text {dust }}=1.2 \times 10^{-27} \mathrm{~kg} \mathrm{~m}^{-3} \cdot s \cdot\left(n^{2}+2 / n^{2}-1\right) \cdot\langle A / L\rangle$

(equation 3.30 in Whittet 1992), where $s$ is the specific density (e.g. $2500 \mathrm{~kg} \mathrm{~m}^{-3}$ for light silicate dust) and $n$ is the refractive index, e.g. 
1.5 for silicates. The local ISM has $\left\langle A / L>=1.8 \mathrm{mag} \mathrm{kpc}^{-1}\right.$, and we use standard values in Whittet (1992): $\rho_{\text {dust }}=2 \times 10^{-24} \mathrm{~kg} \mathrm{~m}^{-3}$ and $\rho_{\text {gas }}=2 \times 10^{-22} \mathrm{~kg} \mathrm{~m}^{-3}$, i.e. with a minimum gas-to-dust ratio of 100 .

In our case, the size of the cloud is $<0.01 \mathrm{pc}$ (rather than typical ISM path lengths of $\mathrm{kpc}$ ), so the dust density we find is $\rho_{\text {dust }}=2 \times$ $10^{-19} \mathrm{~kg} \mathrm{~m}^{-3}$. Thus, $\rho_{\text {gas }}$ is at least $2 \times 10^{-17} \mathrm{~kg} \mathrm{~m}^{-3}$, and $M_{\text {cloud }}=$ $(4 \pi / 3) R_{\text {cloud }}^{3} \rho_{\text {gas }}=0.002 \mathrm{M}_{\odot}$ for $R_{\text {cloud }}=0.01 \mathrm{pc}$. This corresponds to a column density $N_{\mathrm{H}} 10^{21} \mathrm{~cm}^{-2}$. Assuming the gas is cold, as required to prevent sublimation of dust, this is sufficient to imprint a substantial, narrow absorption feature on to the hydrogen emission lines, which is not observed (see e.g. Rees, Netzer \& Ferland 1989).

Here, we have assumed standard ISM dust to get an order of magnitude estimate for the mass required to produce our total extinction; however, as the Guo et al. (2016, fig. 6) extinction curve clearly shows, we are not looking at standard ISM dust. Indeed, as those authors note, there are no identical extinction curves in the literature, but the shape as a function of wavelength that suggests extremely small scatterers. We note that although a very unusual cloud might be able to explain our continuum extinction, given the observed rest frame $\approx$ weeks-long time-scales, the same scenario could not explain the $\mathbf{J} 2317+0005$ event (despite the similar extinction curve).

\subsection{Scenario II: Accretion disc model}

Having discounted an obscuring event as the explanation for J11000053, we turn to accretion disc models. We consider 'cold' accretion flows, described as optically thick, geometrically thin and which drive relatively high-mass accretion rates. They are 'cold' in the sense that the virial temperature of particles near the black hole is low. Similarly, we characterize optically thin, geometrically thick and low-mass accretion rate flows as virially 'hot' accretion flows. Yuan \& Narayan (2014) present a detailed review on virially hot accretion flows around black holes.

After giving our model set-up, we discuss whether J1100-0053 can be described by a 'hot' accretion flow, such as the advectiondominated accretion flow. We then discuss our preferred 'cold' accretion flow model, but where the temperature of the accretion disc is perturbed by propagating cooling and heating fronts in the inner parts $\left(\leq 1000 r_{\mathrm{g}}\right)$ of the accretion disc. Our disc remains virially cold throughout this cycle.

We start with a multitemperature blackbody (MTB) model, with an $L \propto T^{4}$ dependence and a $T \propto r^{-3 / 4}$ relation. A thin accretion disc has a negligible radial pressure gradient. Therefore, at each radius $R$ the gas orbits at the Keplerian angular frequency, $\Omega_{\mathrm{K}}=\left(G M / r^{3}\right)^{1 / 2}$, where $M$ is the mass of the central object, which possesses specific angular momentum $l=\sqrt{G M r}$.

We present in Table 3 the salient details of the models we now present and discuss below.

Zimmerman et al. (2005) compare models with 'zero' and 'nonzero' torque at the ISCO, and the impact on the temperature profile of the corresponding accretion disc when the torque changes. From Zimmerman et al. (2005), the zero torque (ZT) luminosity is given by

$L_{\mathrm{disc}}=\frac{G M \dot{M}}{2 r_{\mathrm{in}}}=73.9 \sigma\left(\frac{T_{\max }}{f}\right)^{4} r_{\mathrm{in}}^{2}$,

and the standard, non-zero torque (NZT) luminosity is given by

$L_{\mathrm{disc}}=\frac{3 G M \dot{M}}{2 r_{\mathrm{in}}}=12.6 \sigma\left(\frac{T_{\max }}{f}\right)^{4} r_{\mathrm{in}}^{2}$, where $f$ is a spectral hardening factor, with a canonical blackbody spectrum having $f=1$ and $\sigma$ is the Stefan-Boltzmann constant. In zero-torque models, the temperature $T_{\mathrm{ZT}}$ goes to zero at the inner edge of the disc (since the torque vanishes there), whereas for a nonzero torque temperature profile, $T_{\mathrm{NZT}}$ reaches its maximum value at the inner edge of the disc (where the torque is maximal). Given an MTB model for disc emission, these differences at small $r_{\mathrm{g}}$ translate to large differences in the SED.

Since we are concerned with inner parts of the disc, we should consider relativistic effects. The ISCO probably lies at $r$ $<6 G M / c^{2}=3 r_{\mathrm{s}}$ since quasar black holes generally have prograde rotation (e.g. Reynolds 2014; Capellupo et al. 2016). The specific binding energy at the ISCO is therefore probably larger than $1-\sqrt{8 / 9} \approx 0.057$, so that even if there were no torque at the ISCO, the ratio of the bolometric luminosity to the monochromatic luminosity at any point in the rest-frame UV/optical spectra could be larger than in a steadily accreting thin disc. We note that since $l=\sqrt{G M r}$, we do not expect the ISCO to change on human time-scales.

As shown in Fig. 3, the SDSS spectrum from 2000 is well fit with a thin Shakura \& Sunyaev (1973) $\alpha$-disc and the NZT condition. However switching to just the zero-torque condition, while suppressing the bluer disc emissivity, is not sufficient to explain the 2010 spectrum. We note that we could have chosen a different normalization in Fig. 3, such that the ZT model would fit our 2000 spectrum; however, maintaining such a normalization would mean the ZT-abs and ZT-Spartfit models would lie above our later spectra.

\subsubsection{Switching states to an RIAF/ADAFs}

A possible explanation for the behaviour of J1100-0053 is that it switches accretion modes, from a virially cold, high $\dot{M}$ flow to a virially hot, low $\dot{M}$ flow. The latter could be either a radiatively inefficient accretion flow (RIAF; Narayan, Mahadevan \& Quataert 1998; Quataert 2001) or an advection-dominated accretion flow (ADAF; Yuan \& Narayan 2014, and references therein).

There are examples of this type of behaviour in lower-luminosity objects. For example, Nemmen et al. (2006) successfully explain the SED for the low-ionization nuclear emission-line region of NGC 1097 with a model where the inner part of the flow is a virially hot RIAF, and the outer part is a standard virially cold thin disc. The broad-band spectrum of NGC 1097 from Nemmen et al. (2006) initially appears similar to the UV/optical 2010 spectrum of J1100-0053. Nemmen et al. (2006, fig. 4) shows the MTB-like model component from the thin disc at $r>225 r_{\mathrm{g}}$ dramatically decreasing at $\sim 10^{15} \mathrm{~Hz}(\sim 300 \mathrm{~nm})$. Nemmen et al. (2006) model the disc region interior to this as an $\operatorname{RIAF}^{6}$ at a power (in $v L_{v}$ ), an order of magnitude lower than the MTB in the optical, but spanning from the X-ray to the far-IR.

Can J1100-0053 switch states from a thin disc quasar to an ADAF at small radii with the thin disc surviving at large radii? Assuming the transition happens due to a thermal instability in the inner disc on the thermal time-scale, and propagates outwards to radii $\sim 225 r_{\mathrm{g}}$ as in Nemmen et al. (2006), we can parametrize the front propagation time as

$t_{\text {front }} \sim 5 \mathrm{yr}\left(\frac{h / r}{0.1}\right)^{-1}\left(\frac{\alpha}{0.3}\right)^{-1}\left(\frac{r}{225 r_{\mathrm{g}}}\right)^{3 / 2} \frac{r_{\mathrm{g}}}{c}$,

\footnotetext{
${ }^{6} \mathrm{~A}$ change to an ADAF is also possible in this model.
} 
Table 3. Salient details of the models presented in Figs 2 and 3.

\begin{tabular}{lllll}
\hline Model & Torque at ISCO & AD suppression & Reddening law & Shown by \\
\hline NZT & $\checkmark$ & $\times$ & $\times$ & Fig. 2 blue solid; Fig. 3 black solid \\
ZT & $\times$ & $\times$ & $\times$ & Fig. 3 black dashed \\
ZT-abs & $\times$ & $\times$ & Fit to Guo et al. (2016) & Fig. 2 red long dashed \\
ZT-Spartfit $_{\text {ZT-Snofit }}{ }^{a}$ & $\times$ & 90 per cent inside $40 r_{g}$ & $\times$ & Fig. 2 red dotted \\
BB(7800K) & $\times$ & 100 per cent inside $225 r_{g}$ & $\times$ & Fig. 3 black dotted \\
\hline
\end{tabular}

Note. We show the ZT-Snofit and BB models only to demonstrate the inability of grey-body absorption to fit the shape of the observed spectra even in the most extreme circumstances. We model the Guo et al. absorption as a piecewise three-line fit to the extinction data in their fig. 6 . The transmission fraction at that wavelength (in $\mathrm{nm}$ ): $\lambda_{\text {break }}=[714.0,435.0,370.0,285.0]$, ext $\mathrm{norm}=[1.0,0.87,0.70,0.25] . \alpha$ is assumed to be 0.3 in all instances.

${ }^{a}$ The ZT-Snofit and BB models are arbitrarily normalized to match the peak of the 2010 spectrum; the other models possess the same relative normalizations.

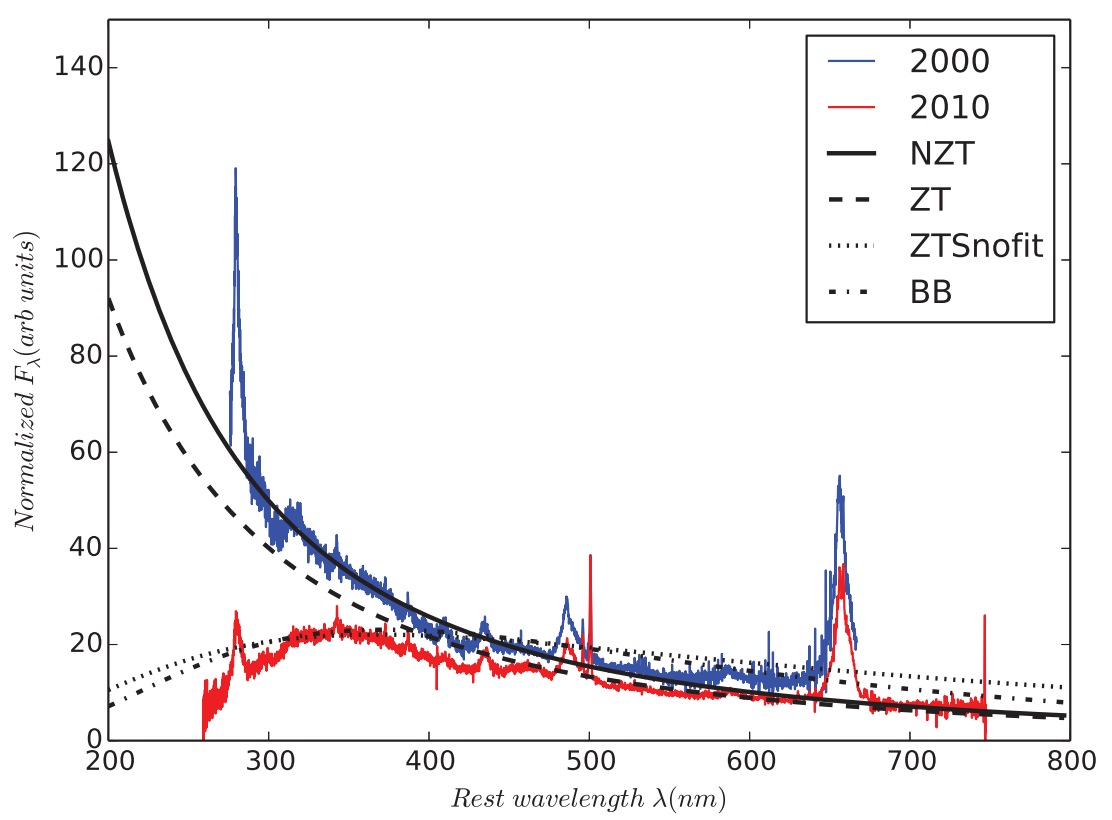

Figure 3. J1100-0053 data (blue line 2000 spectrum; red line 2010 spectrum) with NZT model and three models providing poor fits. The solid black line shows non-zero torque at ISCO (following Afshordi \& Paczyński 2003) and as in Fig. 2, while the dashed black line shows a zero torque at the ISCO model. As noted in the text, a simple shift from a non-zero torque to a zero-torque solution is insufficient to reproduce the 2010 spectrum. Indeed, no form of grey-body absorption can explain the 2010 data, and we plot two example efforts - the dotted line is the continuum from the same zero-torque model but with no emission from inside $225 r_{\mathrm{g}}$, normalized to the peak of the 2010 data. The dash-dotted line is a single blackbody spectrum for $T=7800 \mathrm{~K}$, again normalized to the 2010 peak. We note that though the temperature profile can be altered to produce a 'turnover' in the neighbourhood of $350 \mathrm{~nm}$, the shape of a blackbody is too broad. Worse, in this figure we have arbitrarily enhanced the bolometric luminosity expected from such a source, relative to the NZT and ZT models, as well as the models plotted in Fig. 2 (which all possess the same normalization).

where we have had to assume a higher value of $\alpha \sim 0.3$ (King, Pringle \& Livio 2007) than typically assumed for thin $(h / r \ll 1)$ discs. This is plausible if there exists a very viscous disc and the effect propagates outwards on a time-scale of $\leq 5 \mathrm{yr}$ from the inner disc.

If the viscous disc switches to an RIAF at radii $<225 r_{\mathrm{g}}$, then the UV/optical emission should be suppressed by several orders of magnitude compared to a radiatively efficient thin disc (Narayan et al. 1998; Abramowicz et al. 2002; Abramowicz \& Fragile 2013). However, if the thin disc emission is simply uniformly suppressed within $<225 r_{\mathrm{g}}$ by a large factor, we cannot reproduce the shape of the J1100-0053 spectrum in 2010 (see Fig. 3). Furthermore, in order to restore the thin disc in the 2016 observation, a thermal instability is required to occur at $\sim 225 r_{g}$ and a front to propagate inwards, collapsing the RIAF back to a thin disc.

Noting RIAFs/ADAFs exist at lower luminosity than for a classic thin disc $\left(\epsilon \sim 0.005\right.$ and $\epsilon \sim 0.1$, respectively, for $\left.L=\epsilon \dot{M} c^{2}\right)$ it is unclear first what physical processes would trigger the change of state to an ADAF and then cool back down to a thin disc, and second, why such an instability would occur at the thin disc/RIAF boundary in J1100-0053, whereas in NGC 1097 this interface appears to be stable. In any case, suppressing the MTB temperature profile inside a radius of $225 r_{\mathrm{g}}$ would lead to a collapse in the total flux compared to unperturbed disc. These scenarios are difficult to reconcile with our data.

\subsubsection{Changes at the ISCO and a cold absorbing phase}

An alternative explanation of our observations involves a triggering event at the ISCO and an associated cold, absorbing or scattering phase. Here, we discuss the phenomenological requirements of this model from the photometry in Fig. 1, the spectra in Fig. 2 and the line measurements from Table 1 . First, we outline the model fits 
that are required and then we attempt to construct a simple coherent phenomenological picture.

The SDSS spectrum from 2000 can be well fit with a simple standard thin accretion disc model with non-zero torque at the ISCO. The 2010 spectrum is relatively well fit with a thin disc model but now with zero torque at the ISCO and with a cold absorbing screen very similar to that observed by Guo et al. (2016). Both of these fits are shown in Fig. 3. From Fig. 1, the PanSTARRS fluxes drop strongly in 2011, particularly in the green band, and remain low until about 2014 (MJD 56800), whereupon the DECaLS green and then red fluxes climb back upwards. By 2017, the spectrum is well fit with a zero-torque disc model with strong grey-body suppression of the flux to 10 per cent of an unperturbed disc at radii interior to $40 r_{\mathrm{g}}$. This can be interpreted as a modest change in the effective temperature in the innermost disc at $\leq 40 r_{\mathrm{g}}$. During this time, the WISE $W 1$ and W2 flux reaches a minimum around MJD 57000 (2015) and then climbs back towards the values from 2010. The line measurements in Table 1 imply that the broad lines are stronger in 2010 than in 2017.

Putting all this together into a simple phenomenological model suggests the following course of events, which we depict in cartoon form in Fig. 4. Conditions change around 2010 at the ISCO and this change is associated with a cold absorbing or scattering phase. Since the broad lines are relatively strong in 2010, the change cannot have occurred more than several months before the 2010 spectrum was taken. The triggering event may be a change in $\dot{M}$ due to a stochastic variation in the mass supply or a local change in $\alpha$. Since accretion disc luminosity is probably powered by magnetized gas losing angular momentum, magnetized gas in the plunging region might be expected to torque the ISCO gas (e.g. Gammie 1999; Agol \& Krolik 2000). If there is a change in the magnetic field configuration around the $\mathrm{SMBH}$, such that the torque decreases to near zero at the ISCO, the temperature of the innermost disc will drop dramatically (Cao \& Xu 2003). We speculate that just such a change occurred not long before the 2010 spectrum was taken.

Associated with a change at the ISCO, we require a cold, absorbing and/or scattering phase. There are several possible sources of such a cold phase. A scattering phase might arise from the collapse of a corona. Or a cold phase could appear in the helical outflowing wind that generates the innermost BLR. A dramatic change in disc surface opacity might also be responsible, or some combination of all three of these. So while in 2000 only one model component is needed to fit the spectrum, namely a thin disc with non-zero torque at the ISCO, in 2010 two model components are required: a thin disc with zero-torque at the ISCO and a cold absorbing/scattering screen. The 2010 spectrum cannot be fit with any variety of grey body absorption, or equivalently a simple MTB model with an alternate temperature profile. It is, however, reasonably well fit using the wavelength-dependent absorption model of Guo et al. (2016).

The photometric data in Fig. 1 require a further drop of around a magnitude in the PanSTARRS colours by 2011. We can achieve this by keeping the two-component model from 2010, but by adding a third component, a grey body suppression out to $\sim 100 r_{\mathrm{g}}$. We speculate that a cooling front propagates outwards in the disc from the changes at the ISCO in 2010 so that regions of the disc that contribute strongly to green and red emission are suppressed by 2011. A cooling front propagates at speed $v_{\text {front }}=\alpha c_{\mathrm{s}}$ (Hameury et al. 2009) so $\alpha \geq 0.1\left(c_{\mathrm{s}} / 10^{4} \mathrm{~km} \mathrm{~s}^{-1}\right)$ is required, but with $v_{\text {front }}$ slowing the farther it travels since $c_{\mathrm{s}}$ is expected to fall rapidly with increasing radius (Sirko \& Goodman 2003).

By 2014, the PanSTARRS green and red fluxes begin recovery. This may be associated with the inward propagation of a heating

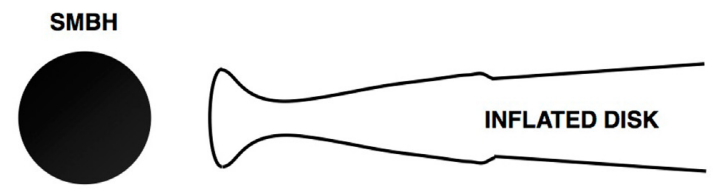

2000

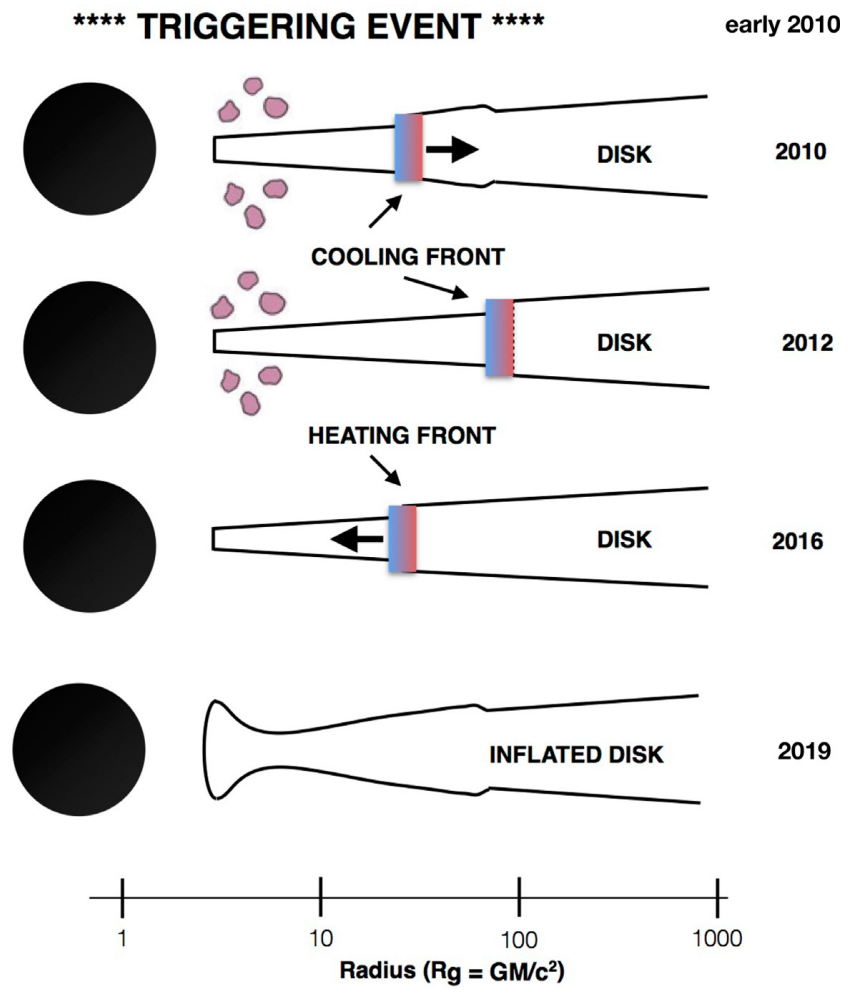

Figure 4. Cartoon illustration of our model explaining the unusual spectral evolution of J1100-0053. In 2000, corresponding to the SDSS spectral epoch, the quasar has a standard inflated accretion disc, i.e. where non-zero torque at the ISCO heats the inner radii of the accretion disc, causing it to puff up (e.g. Zimmerman et al. 2005). Just before the 2010 spectrum, a triggering event occurs that deflates the inner disc, possibly due to a shift in the magnetic field configuration leading to zero torque at the ISCO. This event is associated with some scattering/absorbing cold phase clouds, and causes a cooling front to propagate outwards in the accretion disc, traveling on the $t_{\text {front }}$ time-scale (see also Hameury et al. 2009). Circa 2014, a heating front travels radially inwards, re-heating the inner accretion disc but on longer time-scales, due to the thinner disc. We predict that in the next year, the quasar should roughly return to its initial state.

instability (Hameury et al. 2009). For example, if the surface density, $\Sigma$, reaches a critical value, a heating front can propagate back inwards, analogous to the well-known accretion disc limit cycle mechanism in models of dwarf nova outbursts (e.g. Cannizzo 1998). The returning heating front travels more slowly because the disc is colder and thinner, and $t_{\text {front }}$ is inversely proportional to $h / r$. However, the heating front will re-inflate the disc, as it propagates inwards towards the SMBH. As a result, the recovery to 2010 fluxes in the PanSTARRS bands will take longer than the drop from 2010 to 2011 .

While the cooling/heating has been going on in the accretion disc, the initial dimming of the central ionizing flux will have an effect on the distant (parsec-scale) torus. After a light travel time of $\sim 3 \mathrm{yr}$ the IR flux from the torus should drop (Koshida 2014; Jun et al. 2015b) beginning in 2013, dropping to a minimum in 2014-2015 (since the disc is most suppressed in 2011 and the PanSTARRS 
band recover slightly or remain flat in 2012-2013). We speculate that the absorbing screen has dissipated by this epoch in order to match the IR minimum in 2015. This seems to be consistent with the behaviour of the source in the WISE W1 and W2 bands. The cooling/heating front disc propagation, the connection and origin of the BLR, and the effect on the inner dust have also recently been explored in Baskin \& Laor (2018).

Assuming that nothing else drastic happens, our model predicts that the heating front returns to the ISCO in late 2018 (around now). That means the broad Balmer lines will reach a maximum a few months later in early 2019, but the IR flux should not return to full flux until 2021.

If the cold phase is associated with the condensation or disappearance of part of the inner BLR, then the maximum EWs in 2019 may still be less than the EWs measured in 2010. We also note that if $\mathrm{J} 1100-0053$ is an 'anomalous $\mathrm{H} \beta$ quasar' as suggested by Steinhardt \& Silverman (2013) and described above, then the variable linewidths can be explained by the disc going into a low state and the innermost, fastest, outflow disappears (due to, e.g., reduced radiation pressure). However, we have not been able to constrain the behaviour of $\mathrm{H} \beta$ in $\mathrm{J} 2317+0005$ during the downturn observed by Guo et al. (2016). Therefore, it is not clear whether there is a general link between a broad $\mathrm{H} \beta$ component and an apparent state change in the CLQ disc. As such, we note this potentially intriguing behaviour of the $\mathrm{H} \beta$ emission line and look to investigate further in the context of the 'changing-look' phenomenon in a future demographic study.

By 2017, the returning heating front has propagated back in to $\sim 40 r_{\mathrm{g}}$ and the cold phase has disappeared. So it seems the cold phase is required between 2010 and 2013, but then has fully disappeared by 2017. This is an important constraint on the nature of the cold phase that is an important but poorly understood component in our phenomenological model. If, for example, the cold phase clumps on size scales of order $r_{\mathrm{g}}$, with an overdensity of $10^{6}$ relative to their hotter surroundings, and a relative velocity compared to the hot phase on the order of the orbital velocity, then the clumps are unstable to the Kelvin-Helmholtz instability (equation 3) on an approximate time-scale of

$t_{\mathrm{cc}} \approx 3 \mathrm{mo}\left(\frac{\rho_{\text {cloud }} / \rho_{\text {medium }}}{10^{6}}\right)^{1 / 2}\left(\frac{r_{\text {cloud }}}{r_{\mathrm{g}}}\right)\left(\frac{v_{\text {rel }}}{10^{4} \mathrm{~km} \mathrm{~s}^{-1}}\right)^{-1}$.

The important point here is that cold-phase clouds are unstable to collapse after a short time unless they are extremely overdense with very cold cores relative to the surrounding medium. The main coolants at low temperatures are carbon and oxygen resonance lines and hydrogen and helium from neutral phase material (see e.g. fig. 18 in Sutherland \& Dopita 1993). The ionization energies for carbon and oxygen are 11.26 and $13.61 \mathrm{eV}$, respectively, i.e. $\sim 100 \mathrm{~nm}$, and hence at wavelengths $<100 \mathrm{~nm}$ the disc opacity will increase dramatically in edges. In the inner gas disc such edges must be pressure, turbulence and Doppler broadened, but to depress the flux out to $\sim 300 \mathrm{~nm}$ would require associated velocities of $\sim 0.9 \mathrm{c}$. Thus, cooling absorption edges could explain the cold absorbing phase only if substantial blanketing material is flung out from near the event horizon, but this would not match the mild, longer wavelength absorption required in Guo et al. (2016) or our source. Rayleigh scattering might also help explain the steep cutoff in the 2010 spectrum; we might expect an additional scattering layer from slightly larger ions or neutral atoms in a cold or condensing phase from the corona or the BLR. However, we cannot take a strong position on the identity of the absorber without more data, especially time resolved spectra of an entire event such as this.

\section{CONCLUSIONS}

By monitoring CLQs, we introduce new tests of models of accretion disc physics. We present the quasar J1100-0053 that was catalogued in the SDSS quasar survey, but identified as an interesting due to its IR photometric light curve.

We have shown that a simple phenomenological model with a propagating cooling front is capable of describing the gross spectral and temporal variations in this CLQ. Our model makes a prediction for this source, testable over the next few years and, if confirmed, implies that CLQs as a class are driven by changes near the ISCO, close to the SMBH. The discovery of J1100-0053 (and J2317+0005) are specific key examples of time-domain astronomy and the resulting astrophysics to be studied. However, even with the coverage from WISE, PanSTARRS, SDSS, DECaLS, and CRTS, we have a relatively sparse data set that cannot tightly constrain our theoretical model.

The Zwicky Transient Facility (ZTF; Bellm 2014) has very recently started and will open a new data space with high cadence, multiband photometric monitoring. Along with ZTF in the very near future, the Large Synoptic Survey Telescope (Ivezic \& Tyson 2008; LSST Science Collaborations et al. 2009) will allow identification of the types of events such as J1100-0053 and J2317+0005 while they are occurring, allowing spectroscopic monitoring. We will be able to see how long a UV collapse lasts and closely follow its evolution. Such data will stringently test models of AGN discs at much higher fidelity than we are able to do with current CLQ samples.

Author contributions. NPR led the project, developed and wrote the initial drafts of the manuscript and coordinated the team. KESF and BK originated and developed the theoretical interpretation presented here. MG and DS were heavily responsible for the initial discussions and observations that were the genesis of this project. AMM produced the initial IR variable quasar catalogues. DS, MG, and AJD were part of the Palomar observing team. NPR, AMM, and $\mathrm{AD}$ are part of the DECaLS Legacy Survey. RA, AD, and HDJ contributed to the manuscript.

Availability of Data and computer analysis codes. All materials, data, code, and analysis algorithms are fully available at: https://github.com/d80b2t/WISE_LCs

\section{ACKNOWLEDGEMENTS}

NPR acknowledges support from the STFC and the Ernest Rutherford Fellowship scheme. KESF and BM are supported by NSF PAARE AST-1153335. KESF and BM thank CalTech/JPL for support during sabbatical. MF acknowledges support from NSF grants AST-1518308, AST-1749235, AST-1413600, and NASA grant 16-ADAP16-0232. RJA was supported by FONDECYT grant number 1151408. AMM acknowledges support from NASA ADAP grant NNH17AE75I. HDJ was supported by Basic Science Research Program through the National Research Foundation of Korea (NRF) funded by the Ministry of Education (NRF2017R1A6A3A04005158).

We thank David J. Schlegel for quality checks on the BOSS data, and Chris Done for invigorating discussions at the concept and conclusion of this work. We also thank Giorgio Calderone for discussions on QSFit.

This publication makes use of data products from the WISE, which is a joint project of the University of California, Los Angeles, and the Jet Propulsion Laboratory/California Institute of Technology, and NEOWISE, which is a project of the Jet Propulsion Labo- 
ratory/California Institute of Technology. WISE and NEOWISE are funded by the National Aeronautics and Space Administration.

This research has made use of the NASA/IPAC Extragalactic Database (NED) that is operated by the Jet Propulsion Laboratory, California Institute of Technology, under contract with the National Aeronautics and Space Administration.

This research has made use of data obtained from the SuperCOSMOS Science Archive, prepared and hosted by the Wide Field Astronomy Unit, Institute for Astronomy, University of Edinburgh, which is funded by the UK Science and Technology Facilities Council.

The GALEX GR6/7 Data Release hosted at http://galex.stsci.ed u/GR6/ was used. These data were obtained from the Mikulski Archive for Space Telescopes (MAST). STScI is operated by the Association of Universities for Research in Astronomy, Inc., under NASA contract NAS5-26555. Support for MAST for non-HST data is provided by the NASA Office of Space Science via grant NNX09AF08G and by other grants and contracts.

Funding for SDSS-III has been provided by the Alfred P. Sloan Foundation, the Participating Institutions, the National Science Foundation, and the US Department of Energy Office of Science. The SDSS-III web site is http://www.sdss3.org/. SDSS-III is managed by the Astrophysical Research Consortium for the Participating Institutions of the SDSS-III Collaboration including the University of Arizona, the Brazilian Participation Group, Brookhaven National Laboratory, Carnegie Mellon University, University of Florida, the French Participation Group, the German Participation Group, Harvard University, the Instituto de Astrofisica de Canarias, the Michigan State/Notre Dame/JINA Participation Group, Johns Hopkins University, Lawrence Berkeley National Laboratory, Max Planck Institute for Astrophysics, Max Planck Institute for Extraterrestrial Physics, New Mexico State University, New York University, Ohio State University, Pennsylvania State University, University of Portsmouth, Princeton University, the Spanish Participation Group, University of Tokyo, University of Utah, Vanderbilt University, University of Virginia, University of Washington, and Yale University.

\section{REFERENCES}

Abell G. O., 1959, Leaflet Astron. Soc. Pac., 8, 121

Abramowicz M. A., Fragile P. C., 2013, Living Rev. Relativ., 16, 1

Abramowicz M. A., Igumenshchev I. V., Quataert E., Narayan R., 2002, ApJ, 565, 1101

Afshordi N., Paczyński B., 2003, ApJ, 592, 354

Agol E., Krolik J. H., 2000, ApJ, 528, 161

Aihara H. et al., 2018, PASJ, 70, S8

Alloin D., Pelat D., Phillips M., Whittle M., 1985, ApJ, 288, 205

Antonucci R., 1993, ARA\&A, 31, 473

Appenzeller I. et al., 1998, ApJS, 117, 319

Assef R. J., Stern D., Noirot G., Jun H. D., Cutri R. M., Eisenhardt P. R. M., 2018, ApJS, 234, 23

Bae H.-J., Woo J.-H., 2016, ApJ, 828, 97

Balbus S. A., Hawley J. F., 1991, ApJ, 376, 214

Baskin A., Laor A., 2018, MNRAS, 474, 1970

Becker R. H., White R. L., Helfand D. J., 1995, ApJ, 450, 559

Begelman M. C., McKee C. F., Shields G. A., 1983, ApJ, 271, 70

Bellm E., 2014, in Wozniak P. R., Graham M. J., Mahabal A. A., Seaman R., eds, The Third Hot-wiring the Transient Universe Workshop, SLAC, Stanford. p. 27

Boller T., Freyberg M. J., Trümper J., Haberl F., Voges W., Nandra K., 2016, A\&A, 588, A103

Calderone G., Nicastro L., Ghisellini G., Dotti M., Sbarrato T., Shankar F., Colpi M., 2017, MNRAS, 472, 4051
Cannizzo J. K., 1998, ApJ, 493, 426

Cannon R. D., 1975, Proc. Astron. Soc. Aust., 2, 323

Cannon R. D., 1979, The U.K. 1.2m Schmidt Telescope and the Southern Sky Survey, Royal Observatory, Edinburgh

Cao X., Xu Y.-D., 2003, PASJ, 55, 149

Capellupo D. M., Netzer H., Lira P., Trakhtenbrot B., Mejía-Restrepo J., 2016, MNRAS, 460, 212

Clavel J. et al., 1991, ApJ, 366, 64

Cutri R. M. et al., 2011, Technical report, Explanatory Supplement to the WISE Preliminary Data Release Products, IPAC, Pasadena, USA

Dawson K. et al., 2013, AJ, 145, 10

Dey A. et al., 2018, preprint (arXiv:1804.08657)

Drake A. J. et al., 2009, ApJ, 696, 870

Elvis M. et al., 1994, ApJS, 95, 1

Fukugita M., Ichikawa T., Gunn J. E., Doi M., Shimasaku K., Schneider D. P., 1996, AJ, 111, 1748

Gammie C. F., 1999, ApJ, 522, L57

Guo H., Gu M., 2016, ApJ, 822, 26

Guo H. et al., 2016, ApJ, 826, 186

Hambly N. C. et al., 2001a, MNRAS, 326, 1279

Hambly N. C., Irwin M. J., MacGillivray H. T., 2001b, MNRAS, 326, 1295

Hameury J.-M., Viallet M., Lasota J.-P., 2009, A\&A, 496, 413

Hopkins P. F., 2013, MNRAS, 428, 2840

Ivezic Z. et al., 2008, preprint (arXiv:0805.2366)

Jun H. D. et al., 2015a, ApJ, 806, 109

Jun H. D., Stern D., Graham M. J., Djorgovski S. G., Mainzer A., Cutri R. M., Drake A. J., Mahabal A. A., 2015b, ApJ, 814, L12

Kaiser N. et al., 2010, in Stepp L. M., Gilmozzi R., Hall H. J., eds, Proc. SPIE Conf. Ser. Vol 7733, Ground-based and Airborne Telescopes III. SPIE, Bellingham, p. 77330E

Kepler S. O. et al., 2015, MNRAS, 446, 4078

Kepler S. O. et al., 2016, MNRAS, 455, 3413

King A., 2012, Mem. Soc. Astron. Italiana, 83, 466

King A. R., Pringle J. E., Livio M., 2007, MNRAS, 376, 1740

Koratkar A., Blaes O., 1999, PASP, 111, 1

Koshida S. et al., 2014, ApJ, 788, 159

LaMassa S. M. et al., 2015, ApJ, 800, 144

Lang D., 2014, AJ, 147, 108

Lasota J.-P., 2016, in Bambi C., ed., Astrophysics and Space Science Library Vol. 440, Astrophysics of Black Holes: From Fundamental Aspects to Latest Developments, Springer-Verlag, Berlin. p. 1

Lawrence A., 2012, MNRAS, 423, 451

Lawrence A., 2018, Nature Astron., 2, 102

LSST Science Collaborations et al., 2009, preprint (arXiv:0912.0201)

Lusso E., Risaliti G., 2017, A\&A, 602, A79

MacLeod C. L. et al., 2010, ApJ, 721, 1014

MacLeod C. L. et al., 2016, MNRAS, 457, 389

Magnier E. A. et al., 2013, ApJS, 205, 20

Mahabal A. A. et al., 2011, Bull. Astron. Soc. India, 39, 387

Mainzer A. et al., 2011, ApJ, 731, 53

Mainzer A. et al., 2014, ApJ, 792, 30

Malkan M. A., Sargent W. L. W., 1982, ApJ, 254, 22

Margala D., Kirkby D., Dawson K., Bailey S., Blanton M., Schneider D. P., 2016, ApJ, 831, 157

Martin D. C. et al., 2005, ApJ, 619, L1

McKernan B., Ford K. E. S., Kocsis B., Lyra W., Winter L. M., 2014, MNRAS, 441, 900

Meisner A. M., Bromley B. C., Nugent P. E., Schlegel D. J., Kenyon S. J., Schlafly E. F., Dawson K. S., 2017a, AJ, 153, 65

Meisner A. M., Lang D., Schlegel D. J., 2017b, AJ, 154, 161

Minkowski R. L., Abell G. O., 1963, The National Geographic SocietyPalomar Observatory Sky Survey. Univ. Chicago Press, p. 481

Morgan C. W., Kochanek C. S., Morgan N. D., Falco E. E., 2010, ApJ, 712, 1129

Morgan C. W. et al., 2012, ApJ, 756, 52

Morrissey P. et al., 2007, ApJS, 173, 682 
Mosquera A. M., Kochanek C. S., 2011, ApJ, 738, 96

Nagakura H., Yamada S., 2008, ApJ, 689, 391

Narayan R., Mahadevan R., Quataert E., 1998, in Abramowicz M. A., Björnsson G., Pringle J. E., eds, Theory of Black Hole Accretion Disks. p. 148

Nemmen R. S., Storchi-Bergmann T., Yuan F., Eracleous M., Terashima Y., Wilson A. S., 2006, ApJ, 643, 652

Oke J. B., Gunn J. E., 1983, ApJ, 266, 713

Pâris I. et al., 2017, A\&A, 597, A79

Pereyra N. A., Vanden Berk D. E., Turnshek D. A., Hillier D. J., Wilhite B. C., Kron R. G., Schneider D. P., Brinkmann J., 2006, ApJ, 642, 87

Perlman E., Addison B., Georganopoulos M., Wingert B., Graff P., 2008, Blazar Variability across the Electromagnetic Spectrum. p. 9

Pooley D., Blackburne J. A., Rappaport S., Schechter P. L., 2007, ApJ, 661, 19

Pringle J. E., 1981, ARA\&A, 19, 137

Quataert E., 2001, in Peterson B. M., Pogge R. W., Polidan R. S., eds, ASP Conf. Ser. Vol. 224, Probing the Physics of Active Galactic Nuclei. Astron. Soc. Pac., San Francisco, p. 71

Rees M. J., Netzer H., Ferland G. J., 1989, ApJ, 347, 640

Reynolds C. S., 2014, Space Sci. Rev., 183, 277

Richards G. T. et al., 2002, AJ, 123, 2945

Richards G. T. et al., 2006, ApJS, 166, 470

Risaliti G., Young M., Elvis M., 2009, ApJ, 700, L6

Roig B., Blanton M. R., Ross N. P., 2014, ApJ, 781, 72

Ross N. P. et al., 2012, ApJS, 199, 3

Ruan J. J. et al., 2016, ApJ, 826, 188

Rumbaugh N. et al., 2018, ApJ, 854, 160

Runnoe J. C. et al., 2016, MNRAS, 455, 1691

Schneider D. P. et al., 2002, AJ, 123, 567

Schneider D. P. et al., 2007, AJ, 134, 102

Sesar B., Stuart J. S., Ivezić Ž., Morgan D. P., Becker A. C., Woźniak P., 2011, AJ, 142, 190
Shakura N. I., Sunyaev R. A., 1973, A\&A, 24, 337

Shen Y. et al., 2011, ApJS, 194, 45

Shields G. A., 1978, Nature, 272, 706

Shiokawa H., Krolik J. H., Cheng R. M., Piran T., Noble S. C., 2015, ApJ, 804,85

Sirko E., Goodman J., 2003, MNRAS, 341, 501

Steinhardt C. L., Silverman J. D., 2013, PASJ, 65, 82

Stern D. et al., 2018, ApJ, preprint (arXiv:1805.06920)

Stoughton C. et al., 2002, AJ, 123, 485

Stubbs C. W., Doherty P., Cramer C., Narayan G., Brown Y. J., Lykke K. R., Woodward J. T., Tonry J. L., 2010, ApJS, 191, 376

Sutherland R. S., Dopita M. A., 1993, ApJS, 88, 253

Tonry J. L. et al., 2012, ApJ, 750, 99

Ulrich M.-H., Maraschi L., Urry C. M., 1997, ARA\&A, 35, 445

Vanden Berk D. E. et al., 2004, ApJ, 601, 692

Voges W. et al., 1999, A\&A, 349, 389

Whittet D. C. B., 1992, Dust in the Galactic Environment

Wright E. L. et al., 2010, AJ, 140, 1868

Yang Q. et al., 2018, Astrophys. J., 862, 109

Yuan F., Narayan R., 2014, ARA\&A, 52, 529

Zimmerman E. R., Narayan R., McClintock J. E., Miller J. M., 2005, ApJ, 618,832

\section{SUPPORTING INFORMATION}

Supplementary data are available at https://github.com/d80b2t/WI SE_LCs online.

This paper has been typeset from a $\mathrm{T}_{\mathrm{E}} \mathrm{X} / \mathrm{L} \mathrm{T} \mathrm{E} \mathrm{X}$ file prepared by the author. 